\title{
STAATSRECHTSDENKEN UND VERFASSUNGSVERGLEICHUNG IN MEXIKO
}

\author{
Von Hans-Rudolf HoRn
}

Die mexikanische Verfassungsrechtslehre ist seit ihren Anfängen durch die Fähigkeit gekennzeichnet, die wesentlichen politischen Leitmotive der Zeit aufzugreifen und schöpferisch auszugestalten. Ihre Beiträge werden in der Neuen Welt ${ }^{1}$ mit besonderer Aufmerksamkeit verfolgt; sie haben nicht selten die Entwicklung in anderen lateinamerikanischen Ländern maßgebend beeinflußt. Daher ist es erstaunlich, daß der Erfahrungsaustausch auf dem Gebiet des Verfassungsrechts mit den „repúblicas hermanas“, wie bei offiziellen Begrüßungsansprachen gern gesagt wird, erst in jüngster Zeit erheblich verstärkt und auf eine institutionelle interamerikanische Basis gestellt wurde. Die erfolgreichen Bemühungen in dieser Richtung verdienen prinzipielles Interesse, weil sie über entscheidende Entwicklungslinien von Verfassung und Recht in Übersee Aufschluß geben können.

Die Rechtsvergleichung ist in besonderem Maße die Aufgabe des 1940 an der UNAM (Universidad Nacional Autónoma de México) gegründeten Instituts, das zunächst Instituto de Derecho Comparado de México hieß und das 1968 unter seinem gegenwärtigen Leiter Héctor Fix Zamudio den Namen Instituto de Investigaciones Jurídicas erhielt. Dieses führt gegenwärtig auch die Geschäfte einer anderen interamerikanischen Einrichtung, des Instituto Iberoamericano de Derecho Constitucional, das in Buenos Aires am 22. März 1974 unter Mitwirkung von Staatsrechtslehrern aus den meisten lateinamerikanischen Staaten gegründet wurde. Damit wurde zugleich der organisatorische Rahmen für den Ersten Lateinamerikanischen Kongreß für Verfassungsrecht geschaffen, der im Jahre 1975 in Mexiko-Stadt stattfand. Die interamerikanische Verfassungsdiskussion wurde im März 1976 in Oaxtepec bei Cuernavaca in einem Kolloquium über die Verfassungsentwicklung in den letzten 25 Jahren fortgesetzt. Zum besseren Verständnis dieser beiden Ereignisse ist es zweckmäßig, die wichtigsten Entwicklungslinien des mexikanischen Staatsrechts („derecho constitucional“) zu skizzieren und in einem kurzen Überblick schwerpunktmäßig vor allem zwei Werke aus der Literatur der Gegenwart zu erörtern, weil sie Themen von allgemeinem Interesse behandeln (I). Sodann werden die verfassungsvergleichenden Diskussionen auf dem Ersten Lateinamerikanischen Verfassungsrechts-Kongreß (II) und dem Kolloquium „Evolución de la organización política-constitucional en América Latina 1950-1975“ (III) in den wesentlichen Grundzügen beschrieben. Das Verhältnis von Verfassungsrecht und politischer Wirklichkeit ist Gegenstand einiger Bemerkungen, die sich aus den vorangegangenen Erörterungen ergeben (IV).

\footnotetext{
1 Die beste Bibliographie zur gesamten mexikanischen Rechtswissenschaft wurde von der Kongreßbibliothek der Vereinigten Staaten von Amerika zum ersten Mal 1945 veranlaßt. Die neueste Auflage von "A Revised Guide to the Law and Legal Literature of Mexiko" von Helen L. Clagett und David M. Valderrama wurde 1973 veröffentlicht. Einen ausgezeichneten Ưberblick über die juristische Literatur des letzten Jahrhunderts geben Héctor Fix-Zamudio und Eugenio Hurtado-Marquez mit "La ciencia del derecho en el último siglo: México ${ }^{\alpha}$ in: M. Rotondi, Inchieste di diritto comparato - 6 -, Mailand 1976, S. $461-501$.
} 


\section{Entwicklungslinien des mexikanischen Staatsrechtsdenkens}

Die Gründung des Instituts für Rechtsvergleichung an der UNAM geht zurück auf den spanischen Emigranten Felipe Sánchez Roman. Wie sein Landsmann Manuel Pedroso $^{2}$, der die akademische Lehrveranstaltung des Seminars an der UNAM einführte, hat auch Felipe Sánchez Roman weniger durch seine Veröffentlichungen als durch seine Ausstrahlung als Lehrer das juristische Denken maßgebend beeinflußt. Der unmittelbare Einfluß spanischen Denkens verstärkte sich damals wegen der großen Zahl von Universitätslehrern und Intellektuellen, die infolge des spanischen Bürgerkriegs ihr Land verlassen und in Mexiko Zuflucht gefunden hatten.

Schon in der Zeit unmittelbar nach der Loslösung von Spanien spielten neben dem starken nordamerikanischen Einfluß stets auch Vorbilder aus dem ehemaligen Mutterland eine wichtige Rolle. Unverkennbar sind bei der ersten republikanischen Verfassung von 1824 die Auswirkungen der Verfassung von Cádiz, die 1812 von den Delegierten aus dem gesamten damaligen spanischen Weltreich verabschiedet wurde, darunter auch von insgesamt zwölf Delegierten aus dem Gebiet des heutigen Mexiko. Noch heute enthält die mexikanische Verfassung Regelungen, die aus der Cádiz-Verfassung stammen, wie etwa die Einrichtung der Ständigen Kommission, die im Text von 1812 „Diputación permanente“ (Artikel 157) und in der heutigen Bundesverfassung "Comisión permanente" (Artikel 78 und 79 der Verfassung von 1917) heißt. Umgekehrt läßt sich auch eindeutig ein mexikanischer Einfluß auf die spanische Verfassung von 1931 feststellen insofern, als sie wesentliche Gedanken des Amparo-Verfahrens übernommen hat ${ }^{3}$.

Was den nordamerikanischen Einfluß betrifft, so war auch die Verfassung der Vereinigten Staaten von Amerika aus dem Jahre 1787 Vorbild für die erste föderalistische Verfassung von 1824, die nur für kurze Zeiten durch die unitarischen Verfassungen von 1836 und 1843 abgelöst wurde. Die bundesstaatliche Komponente wurde 1857 erneut bekräftigt und prägt auch die geltende Verfassung von 1917. Der Einfluß des nördlichen Nachbarn wurde nicht zuletzt auch durch das Werk von Alexis de Tocqueville gefördert. Die spanische Übersetzung von „La Démocratie en Amérique" war bereits 1837 in Mexiko bekannt".

Die mexikanische Verfassungsentwicklung weist aber auch bedeutsame eigenständige Errungenschaften auf. Abgesehen von den sozialen Grundrechten der Verfassung von 19175, die weniger ein Verdienst mexikanischer Verfassungsjuristen waren, ist hier vor allem das Amparo-Verfahren zu nennen, das frühzeitig Elemente einer Verfassungsbeschwerde enthielt. Der „juicio de amparo“, zum erstenmal 1841 in der Verfassung des Bundesstaates Yucatán verankert, fand 1857 Aufnahme in die

2 Zur Erinnerung an Don Manuel Pedroso fand im Dezember 1976 in Guanajuato ein internationales Symposion statt, das den 400. Jahrestag der Veröffentlichung der "Six Livres de la République ${ }^{\alpha}$ von Jean Bodin als Ausgangspunkt nahm, vgl. dazu Hans-Rudolf Horn, JZ 1977, 197 f.

3 Jorge Carpizo, La Constitución Mexicana de 1917, 2. Auflage, México D. F. 1973, S. 370.

4 Den berühmten mexikanischen Juristen Ignacio Luis Vallarta, der die Verfassungen der USA und seines eigenen Landes eingehend untersuchte, hat man wegen seines großen Einflusses mit dem Präsidenten des Supreme Court, John Marshal, verglichen, eine Parallele, die allerdings insofern nicht ganz zutreffend ist, als Ignacio Luis Vallarta nur kurze Zeit Präsident des Obersten Gerichtshofes in Mexiko war. Vgl. im einzelnen Felipe Tena Ramírez, Derecho constitucional mexicano, 14. Auflage, México D. F. 1976, S. $85 \mathrm{ff}$.

5 Vgl. dazu Friedrich Wehner, Der mexikanische Präsidialismus und die Verfassung, in diesem Heft S. bes. S. . Zur Entstehung der Vorschriften des Constitucionalismo Social vgl. den Mitautor der einschlägigen Bestimmungen. Pastor Rouaix, Génesis de los artículos 27 y 123 de la Constitución Política de 1917, Puebla, Mexiko, 1945, neu aufgelegt von der Biblioteca del Instituto Nacional de Estudios Históricos de la Revolución Mexicana, México 1959. Vgl. auch Carpizo a. a. O. (s. o. N. 3), S. $117 \mathrm{ff}$. 
Bundesverfassung. Wenn auch sicherlich die Rechtsprechung des amerikanischen Supreme Court, der bereits seit 1801 auch die Verfassungswidrigkeit von Gesetzen prüfte, eine Rolle gespielt haben mag, und sich Vorläufer auch im spanischen Recht, insbesondere des Königreichs Aragón, feststellen lassen, stellt doch der Amparo eine eigenständige Rechtsfigur dar, auf die mexikanische Juristen verständlicherweise recht stolz sind. Ihm widmeten bereits frühzeitig die bedeutendsten Rechtslehrer ihrer Zeit, Ignacio Luis Vallarta ${ }^{6}$ und Emilio Rabasa ${ }^{7}$, eingehende monographische Arbeiten. Beide Autoren waren zugleich Bewunderer des nordamerikanischen Verfassungssystems. ${ }^{8}$

Auch in den letzten Jahrzehnten sind dem Amparo weitere wichtige Arbeiten gewidmet, unter denen das Werk von Ignacio Burgoa ${ }^{9}$ und insbesondere die zahlreichen Veröffentlichungen von Héctor Fix Zamudio hervorzuheben sind ${ }^{10}$. Von seinen Arbeiten liegen nunmehr weitere Auszüge auch in deutscher Sprache vor ${ }^{11}$, nachdem bereits zuvor seine Beiträge in Festschriften und Sammelwerken in der Bundesrepublik Deutschland veröffentlicht worden waren ${ }^{12}$. Sein Werk „Veinticinco años de evolución constitucional 1940-1965“, das anläßlich des fünfundzwanzigjährigen Bestehens des Instituto de Derecho Comparado erschienen ist, stellt ein Panorama der Verfassungskontrolle dar, von dem Karl Loewenstein gesagt hat, daß kein anderes Buch auszumachen sei, wo in rechtsvergleichender Gestaltung umfangreiches Material so handlich zusammengetragen wär ${ }^{13}$.

Zum Verfassungsrecht im umfassenden Sinne liegen aus jüngerer Zeit bedeutende Publikationen vor. Unter den Autoren sind hervorzuheben Ulises Schmill mit „El sistema de la Constitución Mexicana“ (1971) und Ignacio Burgoa, dessen Werk „Derecho constitucional mexicano“ 1973 erschien.

Einige spezielle Leitmotive des mexikanischen Staatsrechtsdenkens können anhand von zwei Werken aufgezeigt werden, die nach Anlage und Aufbau verschieden sind, sich aber gerade deshalb ergänzen. Sie behandeln Fragen, die auch für die Verfassungsdiskussion in der Bundesrepublik Deutschland aktuell sind ${ }^{13 a}$. Die Auto-

6 Ignacio Luis Vallarta, El juicio de amparo y el writ of habeas corpus - ensayo crítico comparativo sobre los recursos constitucionales, México 1881.

7 Emilio Rabasa, El juicio constitucional - origines, teoría y extensión, México 1919.

8 Tena Ramírez a. a. O. (s. o. N. 2), S. 86.

9 Ignacio Burgoa, El juicio de amparo, 9. Auflage México 1974 (erste Auflage 1943); vgl. ferner Mariano Azuela, Introducción al estudio del amparo, Monterrey 1968; Octavio A. Hernández, Curso de amparo, instituciones fundamentales, México 1966; Romeo León Orantes, El juicio de amparo, 3. Auflage Puebla 1957; Luis Bazdresh, Curso elemental del juicio de amparo, Guadalajara, 1972; Salvador Castro Zavaleta, Práctica del juicio de amparo, México 1971; Humberto Briseño Siera, Teoría y técnica del amparo Puebla 1966, und Amparo mexicano, México 1971; Juventino V. Castro, Lecciones de garantías y amparo, 1974; Arturo González Cosío, El juicio de amparo, México 1973; Jorge Trueba Barrera, El juicio de amparo en materia de trabajo, México 1963; Ricardo Couto, La suspensión del acto reclamado en el Amparo, México 1929, unter dem Titel "Tratado teórica-práctico de la suspensión en el amparo, con un estudio sobre la suspensión con efectos de amparo provisional“ 1973 in dritter Auflage erschienen; Ignacio Soto Gordoa und Gilberto Liévana Palma, La suspensión del acto reclamado en el juicio de amparo, México 1959. Zum Amparo-Verfahren in deutscher Sprache vgl. Hans-Rudolf Horn, Das Amparo-Verfahren in Mexiko, VRU 1968, 162, und México: Revolution und Verfassung, Hamburg 1969, S. $83 \mathrm{ff}$; Hans Christoph von Rohr, Der argentinische Amparo-Prozeß unter Berücksichtigung ähnlicher Verfahren in Brasilien, Mexiko und Peru, Bonn 1969; vgl. auch Anm. 11.

10 Héctor Fix-Zamudio, El juicio de amparo, 1964, und Estudio sobre la jurisdicción constitucional mexicana, in: Mauro Cappelletti, $\mathrm{La}$ jurisdicción constitucional de la libertad; con referencia a los ordenamientos alemán, suizo y austriaco, übersetzt von Fix-Zamudio selbst, México 1961, S. 129-247.

11 Héctor Fix-Zamudio, Verfassungskontrolle in Lateinamerika, JöR 1976, 649-693, übersetzt und bearbeitet von Hans-Dieter Nahme und Hans-Rudolf Horn. Eine deutsche Fassung enthielt bereits das Journal der Internationalen Juristen-Kommission, Genf, Band IX Nr. 2 (1968), S. 69-108, Der prozessuale Rechtsschutz des einzelnen in Lateinamerika.

12 Judicial Protection of the Individual against the Executive in Mexico, in: Gerichtsschutz gegen die Exekutive, Band 2, Köln 1969, S. 713-770; Influencia del derecho angloamericano en la protección procesal de los derechos humanos en América Latina, in Festschrift für Karl Loewenstein, Tübingen, 1971, S. $485-508$

13 Vgl. dazu das Vorwort in JöR 1976, $650 \mathrm{f}$.

13a Vgl. dazu den Schlußbericht der Enquête-Kommission „Verfassungsreform ${ }^{\alpha}$, Bundestags-Drucksache $7 / 5924$, S. $11 \mathrm{ff}$., $49 \mathrm{ff}$. veröffentlicht auch vom Presse- und Informationszentrum des deutschen Bundestages in: Zur Sache 3/76 und 2/77. 
ren der beiden Werke, die hier ein wenig eingehender gewürdigt werden sollen, stammen aus verschiedenen Generationen. Felipe Tena Ramírez, ehemaliger Richter am Obersten Gerichtshof und Professor der UNAM, ist seit 1944 Verfasser des klassisch gewordenen Lehrbuches „Derecho constitucional mexicano“, das im Jahre 1976 seine 14. Auflage erreichte. Jorge Carpizo ist dagegen noch ein junger Wissenschaftler; er ist im Instituto de Investigaciones Jurídicas tätig und ist der Verfasser einer 1969 erschienenen Monographie „La Constitución Mexicana de 1917“, die bereits 1973 ihre zweite Auflage erfuhr.

Carpizo stellt besonders die sozialen Grundrechte der mexikanischen Verfassung und ihre Ausstrahlung auf andere Länder in den Vordergrund. Tena Ramírez behandelt diese Fragen allenfalls am Rande, nicht weil er sie für unwichtig hält als vielmehr aus dem äußerlichen Grunde, daß sie Gegenstand eigener Lehrbücher, besonders des Arbeitsrechts und des Agrarrechts, sind.

Beide Autoren befassen sich mit einem "Geburtsfehler" der Verfassung von 1917, den diese im übrigen mit dem Bonner Grundgesetz gemeinsam hat: die Verfassung von Querétaro wurde nicht in einer Volksabstimmung gebilligt ${ }^{14}$. Während aber Tena Ramírez betont, daß dieser Mangel längst durch die Tatsache geheilt ist, daß das Volk seine Zustimmung durch die Befolgung der Verfassung und insbesondere durch Wahlen bekundet hat, kommt Carpizo offenbar schwerer über den Mangel der von ihm postulierten demokratischen Legitimation hinweg. Er steht nämlich auf dem Standpunkt, daß das Prinzip der Repräsentation dem Gedanken der Volkssouveränität zuwiderläuft. Obwohl er wiederholt Carl Schmitt zitiert, übersieht er, daß dieser mit aller Klarheit die inzwischen herrschend gewordene Lehre dargetan hat: die Repräsentation ist ein wesentliches Element einer jeden staatlichen Einheit. Selbst die Vollversammlung aller stimmberechtigten Bürger, die sich nach dem Ideal von Rousseau in einigen Schweizer Kantonen noch zusammenfindet, um Gesetze zu beschließen, ist niemals mit der politischen Einheit identisch, sondern repräsentiert diese ${ }^{15}$. Das Prinzip der Repräsentation muß daher erst recht in unseren Massendemokratien als notwendiges Konstitutionsprinzip anerkannt werden. Tena Ramírez widmet von daher den Problemen des Wahlrechts und der politischen Parteien besondere Aufmerksamkeit.

Wer aber wie Carpizo das System der Repräsentation als undemokratisch ablehnt, kann sich kaum mit der Vielzahl der Verfassungsänderungen abfinden, die inzwischen nach den Vorschriften der Verfassung ohne Befragung der Stimmbürgerschaft stattgefunden haben. Auch Tena Ramírez ${ }^{16}$ sieht die Gefahr, daß durch eine Häufung von Verfassungsänderungen ohne Beteiligung des Volkes eine Entfremdung zwischen diesem und seinen Repräsentanten eintritt. Im Gegensatz zu Carpizo lehnt er aber jede Form eines Referendums oder eines Plebiszites ab; er begnügt sich vielmehr mit dem interessanten Vorschlag, daß eine beabsichtigte Verfassungsänderung jeweils vor einer der alle drei Jahre stattfindenden Wahlen bekanntgegeben und erläutert wird, damit die Meinung der Bevölkerung festgestellt werden kann. Erst nach den Wahlen soll dann der Kongreß die Möglichkeit haben,

14 Tena Ramírez a. a. O., S. 79-81 und Carpizo a. a. O. bes. S. $278 \mathrm{f}$.

15 Carl Schmitt, Verfassungslehre, 4. Auflage, Berlin 1965, S. 206 ff., ebenso Gerhard Leibholz, in: Evangelisches Staatslexikon, Stuttgart und Berlin, 2. Auflage 1975, Spalte $1859 \mathrm{ff}$. und Joseph Kaiser, in: Staatslexikon, 6. Auflage, Band VI, Freiburg 1961, Spalte $865 \mathrm{ff}$. Vgl. neuerdings auch den Schlußbericht der Enquête-Kommission Verfassungsreform, Bundestags-Drucksache 7/5924, S. $11 \mathrm{ff}$.

16 A. a. O., S. $68 \mathrm{ff}$. 
die Verfassungsänderung zu beschließen. Die dadurch bewirkte Erschwerung von Verfassungsänderungen ist bei diesem Vorschlag beabsichtigt.

Beide Autoren äußern sich auch zur Frage der Verfassungsrevision. Seit der ersten Verfassungsänderung vom 8. Juli 1921 bis zum Februar 1976 wurde die Verfassung nicht weniger als $178 \mathrm{mal}$ geändert. Den absoluten Rekord erreicht die Regierungszeit von Präsident Luis Echeverría Alvarez (1970-1976) mit 40 Änderungen des Verfassungstextes; diese Zahl kann als von der Regierungszeit des Präsidenten Plutarco Elías Calles übertroffen gelten nur dann, wenn man zu den 18 Änderungen seiner Amtszeit von 1924 bis 1928 auch noch die Epoche des "maximato“ rechnet, in der drei von Calles, dem „maximo lider de la revolutión“, abhängige Präsidenten regierten (1928-1934); dann fallen insbesondere die weiteren 22 Verfassungsänderungen ins Gewicht, die in der nur wenig mehr als zwei Jahre währenden Präsidentschaft von Abelardo L. Rodríguez (1932-1934) ergingen.

Bei einer derartigen Häufung von Verfassungsänderungen drängt sich die Frage nach deren Legitimität auf. Das Problem der Verfassungswidrigkeit von Verfassungsreformen oder auch von ursprünglichen Verfassungsvorschriften ist, wie es scheint, zuerst in Mexiko erörtert worden, und zwar bereits 1912 von dem bereits erwähnten Emilio Rabasa. In neuerer Zeit folgten weitere Veröffentlichungen. Sie stimmen größtenteils darin überein, daß bestimmte verfassungsrechtliche Grundentscheidungen einer Änderung nicht zugänglich sind ${ }^{17}$. Die erste republikanische Verfassung Mexikos von 1824 enthielt noch das Verbot, die Bestimmungen über die Unabhängigkeit, Religion, Regierungsform, Pressefreiheit, Gewaltenteilung und das Prinzip des Föderalismus zu ändern, während die geltende Verfassung keine dem Artikel 79 Abs. 3 des Bonner Grundgesetzes vergleichbare Bestandsschutzregelung enthält.

Eine weitere verfassungspolitisch äußerst wichtige Frage, die sich einer juristischen Betrachtung weitgehend entzieht, ist das spezifisch mexikanische System einer regierenden Partei, die seit ihrer Gründung durch Calles 1928/29 alle Wahlen auf der Ebene des Bundes, der Staaten und der Gemeinden (von einigen wenigen presidentes municipales abgesehen) gewonnen hat. Tena Ramírez widmet dem Problem der "partido oficial“, wie die Partido Revolucionario Institucional (PRI) allgemein auch von Verfassungsrechtlern genannt wird, eingehende Untersuchungen. Die geltende Verfassung enthält zwar jetzt das Wort „partido“: in Artikel 54 werden „diputados de partido“ angeführt. Damit sind aber gerade nicht die Abgeordneten der Partido Revolucionario Institucional gemeint. Der mexikanische Verfassungsgeber hat nämlich seit 1962 das bisher bestehende reine Mehrheitswahlrecht, das Jahrzehnte hindurch kaum einem Vertreter einer oppositionellen Partei eine Chance ließ, zugunsten der kleineren Parteien modifiziert. Wenn sie eine bestimmte Mindeststimmenzahl erreichen, können sie jetzt bis zu 25 „diputados de partido" in die Abgeordnetenkammer des Bundes entsenden. Trotz ihrer zahlenmäßigen Schwäche ist es der Opposition gelungen, die parlamentarischen Debatten zu beleben.

Gleichwohl bleibt das Problem bestehen, daß die PRI weniger eine politische Partei als einen "parastaatlichen Organismus" darstellt, wie Tena Ramírez sagt ${ }^{18}$, der im übrigen realistisch genug ist, um nicht im Namen der reinen Demokratie das System der "partido oficial“ schlechthin zu verdammen; er räumt ein, daß das Ziel

17 Tena Ramírez a. a. O., S. 57 ff. 
einer wahren Demokratie, wie die Verfassung sie fordert, nicht mit Gewalt und nicht mit einem Satz erreicht werden kann, und erkennt auch an, daß das mexikanische System den Vorzug hat, politische Stabilität und sozialen Frieden im Gefolge gehabt zu haben. Er ist bereit, in einer Fußnote ${ }^{19}$ zuzugeben, daß er seine ursprünglich heftige Kritik „más bien desde el aspecto de la realidad nacional que de la doctrina" seit der 11. Auflage eingeschränkt habe.

Schließlich widmen Carpizo und Tena Ramírez sich auch dem mexikanischen Präsidialsystem. Carpizo beschreibt die ungewöhnlich starke Stellung des mexikanischen Präsidenten, die ihn de facto auch zum Chef der Regierungspartei mache. Der Präsident der Republik nimmt nach den Worten Carpizos die Stelle eines europäischen Königs des 18. Jahrhunderts ein: Er bestimmt während seiner Amtszeit den Lebensstil des Volkes ${ }^{20}$. Tena Ramírez, der sich als Lehrbuchautor stärker auf die verfassungsrechtlichen Befugnisse des Präsidenten konzentriert, tritt demgegenüber dafür ein, die verfassungsmäßigen Rechte des Präsidenten eher noch zu verstärken. Dies widerspricht nur auf den ersten Blick den Ausführungen von Carpizo. Denn Tena Ramírez will in größerem Umfang Rechtssetzungsbefugnisse auf den Präsidenten übertragen und das Parlament von den Regelungen technischer Art entlasten, um so dem Trend der „Abdankung des Parlaments“, wie er es nennt, entgegenzuwirken. Die Abgeordneten sollen die Möglichkeit erhalten, sich auf die politisch bedeutsamen Gesetzgebungsvorhaben zu konzentrieren, während es der Exekutive überlassen bleibt, in größerem Umfang im Verordnungswege die erforderlichen Rechtsvorschriften zu erlassen ${ }^{21}$.

In einer Übersicht über die mexikanische Verfassungsrechtsliteratur bedürfen die rechtsvergleichenden Arbeiten, die dem Instituto de Investigaciones Jurídicas entstammen, besonderer Hervorhebung. In diesem Zusammenhang sind anzuführen David Pantoja Morán, „La idea de soberanía en el constitucionalismo latinoamericano“ (1973), Diego Valadés „La dictadura constitucional en América Latina“ (1974) und José Francisco Ruiz Massieu „Normación constitucional de los partidos políticos en América Latina“ (1972). Auch Jorge Carpizo schrieb 1973 ein Buch über den Föderalismus in Lateinamerika und bereits 1971 „Lineamientos constitucionales de la Commonwealth".

\section{Erster Verfassungsrechts-Kongreß 1975}

Veranstalter des Ersten Lateinamerikanischen Verfassungsrechtskongresses und des Kolloquiums über die Entwicklung des Verfassungsrechts 1950-1975 war neben dem Instituto de Investigaciones Jurídicas, das von der Friedrich-Ebert-Stiftung und deren damaligem Vertreter in Mexiko, Dr. Dieter Koniecki, unterstützt wurde, das Instituto Iberoamericano de Derecho Constitucional. Dessen Präsident ist in Personalunion der Leiter des Instituto de Investigaciones Jurídicas, Héctor Fix Zamudio; geschäftsführender Generalsekretär ist Jorge Carpizo. Im Vorstand sind

18 A. a. O., S. 299. Zum Problem der "offiziellen Partei ${ }^{\alpha}$ vgl. Hans-Rudolf Horn, México: Revolution und Verfassung, 1969, S. 58 ff. und Robert K. Furtak, Revolutionspartei und politische Stabilität in México, Hamburg, 1969, sowie Manfred Mols und Hans Werner Tobler, Mexiko: die institutionalisierte Revolution, Köln-Wien 1976, insbesondere S. $49 \mathrm{ff}$.

19 A. a. O., S. 304, N. 13.

20 Carpizo a. a. O., S. 359.

21 Tena Ramírez a. a. O., S. 242 ff. Zum Problem der Rechtsetzungsbefugnisse der Exekutive vgl. neuerdings auch Humberto J. La Roche, Habilitaciones legislativas en Venezuela, Maracaibo 1976. 
neben den weiteren Mexikanern Diego Valadés und Rolando Tamayo die argentinischen Verfassungsrechtslehrer Vanossi, Frías und Quiroga, aus Brasilien Pinto Ferreira, aus Venezuela Manuel García Pelayo, aus Kolumbien Carlos Sáchica, aus Peru García Belaunde und aus Guatemala Jorge Mario García Laguardia vertreten.

Der Erste Lateinamerikanische Verfassungsrechts-Kongreß fand vom 25. bis 30. August 1975 in Mexiko-Stadt statt. Seine wichtigsten Ergebnisse und Empfehlungen, die durch die UNAM Ende 1975 veröffentlicht wurden unter dem Titel „Derecho y realidad constitucional en América Latina“, sind in der spanischen Urfassung im Anschluß an diesen Bericht abgedruckt. In der Präambel zu den Empfehlungen, in denen die Beratungsergebnisse zusammengefaßt werden, sind bereits mit allem Nachdruck die maßgebenden Befunde der sozialen Wirklichkeit und die Postulate herausgestellt, die sich aus den gegebenen Verhältnissen ergeben. Das Verfassungsrecht kann - wie es weiter heißt - nicht in einem bloß formalen Statut bestehen, sondern muß eine Ordnung zum Gegenstand haben, die der Verwirklichung der sozialen Gerechtigkeit dient.

Die Empfehlungen des Kongresses beziehen sich im einzelnen auf folgende Themenkreise:

1. Die Vorherrschaft der vollziehenden Gewalt,

2. Die Verfassung und die pressure groups,

3. Verfassungsänderungen,

4. Die Funktion der rechtsprechenden Gewalt in den Verfassungssystemen Lateinamerikas.

Zu 1.: Das Übergewicht der Exekutive wird auf politische, wirtschaftliche, soziale und kulturelle Faktoren zurückgeführt. Das verfassungsmäßige, demokratisch legitimierte Präsidialsystem ist zu trennen vom Caudillismus, der Diktatur und anderen pathologischen Formen des Übergewichts der vollziehenden Gewalt. Einen Unterschied zum nordamerikanischen System bildet vor allem das Verbot der Wiederwahl in den meisten lateinamerikanischen Staaten. Die verfassungsmäßige Ordnung ist zu gewährleisten durch eine Verstärkung des Prinzips des Gleichgewichts der Kräfte; Voraussetzungen hierzu sind die Gewaltenteilung und das föderalistische System, aber auch die politische Erziehung und die Meinungs- und Pressefreiheit.

Auffällig ist die These $10 \mathrm{zu}$ Thema 1: Danach soll im Gesetz oder in der Verfassung eine Personalunion zwischen dem Führer der "partido oficial“ und dem Chef der Exekutive verboten sein. Dabei ist weniger der Gegensatz zu traditionsreichen Demokratien wie der englischen erstaunlich, in denen der Premierminister stets der Führer der siegreichen Partei ist, als vielmehr die Selbstverständlichkeit, mit der nicht nur in Mexiko Verfassungsrechtler den Begriff „partido oficial“ gebrauchen. In El Salvador wird er beispielsweise für die „Partido de Conciliación Nacional" benutzt.

$\mathrm{Zu}$ 2.: Die Thesen zum Problem der pressure groups können nur als Ausgangspunkt weiterer Erörterungen angesehen werden. Mängel beim Funktionieren der Verfassungen werden ohne nähere Begründung und die unerläßlichen Konkretisierungen "den regionalen, nationalen und internationalen pressure groups" angelastet.

$\mathrm{Zu}$ 3.: Die Thesen zum Thema "Verfassungsänderungen“ beziehen sich in erster Linie auf das Funktionieren der staatlichen Organe. Den rein organisatorischen Regelungen der Verfassung werden die Normen gegenübergestellt, die Grenzen und 
Inhalt staatlichen Handelns umschreiben. Die Probleme der Beschränkung des Eigentums im sozialen Interesse und der Nationalisierung von Unternehmen, die ausdrücklich gegenüber der Enteignung im überlieferten Sinn abgehoben wird, werden als Fragen dargestellt, die nicht allein nach formalen Kategorien zu erfassen sind. Wir treffen hier auf ähnliche Empfehlungen wie in der UN-Charta der wirtschaftlichen Rechte und Pflichten der Staaten, die im wesentlichen auf den mexikanischen Präsidenten Echeverría (1970-1976) zurückgeht ${ }^{22}$.

$\mathrm{Zu}$ 4.: Bei der Formulierung der Thesen zur Verbesserung des Rechtsschutzes des Staatsbürgers gegenüber dem Staat macht sich die besonders eingehende Vorbereitung bemerkbar, die sich auch auf die Ergebnisse vorangegangener interamerikanischer Kongresse stützen kann. Hier wird auch die persönliche Handschrift des über die Grenzen Lateinamerikas angesehenen Kongreßpräsidenten Fix-Zamudio deutlich, der sich seit Jahren den verfassungsrechtlichen und prozessualen Fragen des Grundrechtsschutzes in internationaler Zusammenarbeit gewidmet hat.

Die wichtigste Forderung ist die Einführung einer eigenständigen Verwaltungsgerichtsbarkeit, die von der Regierung in jeder Hinsicht unabhängig ist und das Recht besitzt, ihre Entscheidungen selbst gegenüber Verwaltungsstellen zu vollstrecken. Als die wichtigsten Hindernisse für ein ordnungsgemäßes Funktionieren der Gerichtsbarkeit, besonders der obersten Gerichte, die über verfassungsrechtliche Fragen zu entscheiden haben, werden klar angesprochen

- die unangemessen weite Auslegung des Begriffes der Regierungsakte, die gerichtlich nicht nachgeprüft werden können,

- die ständige Erklärung eines nationalen Notstandes und

- die Verkündung von Vorschriften, die zu Recht oder zu Unrecht als revolutionär qualifiziert werden.

Zur Abhilfe wird vorgeschlagen, die vor einiger Zeit vom Obersten Argentinischen Gerichtshof mutig verfochtenen Prüfungskriterien der "Vernünftigkeit" das Gericht nimmt damit das Recht in Anspruch, eine getroffene Regelung als unvernünftig abzulehnen - und die vom französischen Conseil d'Etat entwickelte Lehre vom detournement de pouvoir heranzuziehen, um den Ermessensbereich der Exekutive sachgerecht zu begrenzen. Bei der Kontrolle der Verfassungsmäßigkeit von Gesetzen wird gefordert, das nur historisch zu erklärende Prinzip aufzugeben, daß die Entscheidung stets nur für den Einzelfall gilt ${ }^{23}$. Eine allgemein verbindliche Entscheidung über die Verfassungswidrigkeit eines Gesetzes bedeutet eine größere Effektivität der Verfassungsgerichtsbarkeit. Eine Reihe weiterer Vorschläge sollen dazu beitragen, die Unabhängigkeit der Richter sicherzustellen, der Langsamkeit und Schwerfälligkeit des Gerichtsverfahrens zu begegnen und den minderbemittelten Bevölkerungsschichten leichter $\mathrm{zu}$ ihrem Recht $\mathrm{zu}$ verhelfen. Der Richter muß in die Lage versetzt werden, aus der Kenntnis der sozialen Verhältnisse heraus und unter Beachtung der Verfassungsnormen Recht zu sprechen.

22 Deutscher Text abgedruckt im Bericht der Bundesregierung zur Entwicklungspolitik, BT-Drucksache 7/4293 vom 10. 11. 1975, S. $77 \mathrm{ff}$. Vgl. dazu Heinrich Jüttner, Rechtsschutz von Investitionen in Entwicklungsländern, VRU 1976, S. $201 \mathrm{ff}$.

23 Hans-Rudolf Horn, VRƯ 1968, S. 166. 
Die Empfehlungen des Ersten Lateinamerikanischen Verfassungsrechts-Kongresses schließen mit der Hervorhebung des mexikanischen Amparo, der seit mehr als einem Jahrhundert vielen Ländern Lateinamerika als Modell für ein Verfahren gedient hat, das den Staatsbürger wirksam gegen den Mißbrauch staatlicher Gewalt schützt. Im Amparo-Verfahren sind - wie betont wird - allgemeine Begriffe, die für jeden Prozeß gelten, verwirklicht. Hier kommen alle Elemente zusammen, die geeignet sind, einen wirksamen Schutz der Regierten zu gewährleisten.

Der Kongreß umfaßte 6 Hauptvorträge von Rednern aus verschiedenen spanischamerikanischen Staaten und rund 50 Referate. $\mathrm{Zu}$ erwähnen ist darunter das Referat des nordamerikanischen Verfassungsrechtlers J. A. C. Grant, der in einer rechtsvergleichenden Studie Gerichtsverfassungsprobleme in den USA, Argentinien und Mexiko behandelte.

\section{Kolloquium über die Verfassungsentwicklung 1976}

Zur Fortsetzung des Dialogs, der auf dem Ersten Verfassungsrechts-Kongreß 1975 begonnen hatte, kamen am 28. März bis zum 2. April 1976 erneut Staatsrechtslehrer aus fast allen Staaten der Hemisphäre in Mexiko zusammen. Thema der Erörterungen war die verfassungsrechtliche Entwicklung in den letzten 25 Jahren: „Evolución de la organización politico-constitucional en América Latina: 1950 bis 1975.“

Die Fülle des dargebotenen Materials macht die Wiedergabe auch nur der wichtigsten verfassungsrechtlichen Veränderungen in Lateinamerika von 1950-1975 und deren Würdigung unmöglich. Ohne Anspruch auf Vollständigkeit sollen daher nur einige Bemerkungen zu typischen Entwicklungslinien gemacht werden, die im Laufe des Kolloquiums deutlicher wurden.

Besonders ausführlich wurde Argentinien erörtert. Dort setzten sich in dem Berichtszeitraum die politische Instabilität und die sozio-ökonomischen Veränderungen fort, die 1930 begonnen hatten. In den Jahren 1943, 1955, 1962 und 1966 - und 1976 ist hinzuzufügen - führten Staatsstreiche oder andere Bewegungen Militärs an die Macht. Mit Germán Bidart Campos kann man die Epochen der Verfassungsentwicklung wie folgt einteilen: 1. bis 1955 die erste Epoche des Peronismus, der sich im Kampf mit der liberalen Tradition des Landes befand; 2. 1955-1958 der gescheiterte Versuch einer Demokratisierung und „deperonización“; 3. 1958-1966 das mißglückte Unternehmen, einen Teil des Peronismus in einer sozialen Demokratie zu integrieren; 4. das Modernisierungsprogramm 1966-1972, das zu erheblichen Spannungen führt; 5. 1972 und 1973 erneute Bemühungen, die Einheit und Versöhnung des Landes zu erreichen und 6. 1973 bis 1976 die Epoche des zweiten Peronismus.

Uber die einzelnen Epochen berichteten außer Bidart Mario Justo López, Pedro J. Frías, Jorge R. Vanossi und Humberto Quiroga Lavié. Frías widmete ferner dem argentinischen Föderalismus ein besonderes Referat.

Brasilien, das im Gegensatz zu der Verfassungsinflation mancher anderer lateinamerikanischer Staaten nur insgesamt sechs Verfassungen in seiner Geschichte aufzuweisen hat, wahrt in seiner geltenden Verfassung von 1967, wie sein Vertreter Pinto Ferreira darlegte, die traditionellen Grundlagen des republikanischen Bundesstaates, verstärkt aber zugleich die Macht der Union und des Präsidenten der 
Republik. Die Militärgerichtsbarkeit wird erweitert, um die „Ideen der brasilianischen Revolution" zu verteidigen. Im übrigen legt die Verfassung den Akzent auf Entwicklungsprobleme und die Agrarreform. 1969 wurde die Verfassung entscheidend geändert, nachdem es zu schwierigen Konflikten zwischen den Verfassungsorganen gekommen war. Das Ergebnis war ein erheblicher Machtzuwachs für den Präsidenten.

Die verfassungsrechtliche Entwicklung in Brasilien im einzelnen behandelten Referate von Arthur Castro Borges und José Alfonso da Silva, der 1976 sein Lehrbuch "Curso de direito constitucional positivo" veröffentlichte.

Costa Rica, über dessen Verfassungsentwicklung Eduardo Ortiz berichtete, zeichnet sich im Vergleich zu manchem größeren Staat durch eine bewundernswerte politische und soziale Stabilität aus. Freilich hat auch Costa Rica, dessen Verfassung von 1949 einen Kompromiß zwischen der mehrheitlichen konservativen Einstellung und fortschrittlichen Neuerungen darstellt, wirtschaftliche und soziale Probleme. Das Land steht daher vor der Aufgabe, sich weiterhin um ein Gleichgewicht zwischen staatlichen Eingriffen und der Förderung der Privatinitiative und eine bessere Vermögensverteilung zu bemühen, um den Gegensatz zwischen arm und reich zu vermindern.

Venezuela hat, wie Humberto J. La Roche ausführte, seit dem Ende der Diktatur von Perez Jimenez im Jahre 1958 eine demokratische Entwicklung aufzuweisen. Die geltende Verfassung von 1961 verankert das Habeas-Corpus- und das Amparo-Verfahren und führte einen obersten Gerichtshof ein. Bemerkenswert sind ferner einige plebiszitäre Züge wie das Referendum bei einer allgemeinen Verfassungsänderung und das Recht der Gesetzesinitiative, das 20000 Wählern eingeräumt wird.

Politisch und wirtschaftlich ist die Lage in Peru, das Gegenstand des Referats von Domingo Carcía Belaúnde war, und in Chile weniger günstig. Über Chile berichtete Jorge Tapía, der als Emigrant in den USA an der Yale University lehrt. Camilo Mena Mena berichtete über Ecuador, dessen elf Regierungen zwischen 1950 und 1975 nur insgesamt 16 Jahre lang auf verfassungsmäßiger Grundlage amtierten. Gegenstand weiterer Referate waren sodann Kolumbien (Carlos Restrepo Piedrahita und Luis Carlos Sáchica), El Salvador (Luis Dominguez Parada), Guatemala (García Laguardia), Honduras (Roberto Ramírez), Nicaragua (Virgilio Godoy) und Panama (Humerto E. Ricord). Anders als die meisten Referenten konnten die Vertreter Mexikos Einzelprobleme des Verfassungsrechts herausgreifen und zum Gegenstand von gesonderten Referaten machen. So behandelte Jorge Carpizo die Stellung der Exekutive und beleuchtete die Nominierung des Präsidentschaftskandidaten der PRI. Diego Valadés erörterte eingehend Fragen des Parlaments und der Oppostion, Fix-Zamudio die rechtsprechende Gewalt ${ }^{24}$, Ignacio Carrillo Prieto die Entwicklung des Arbeits- und Sozialrechts, Manuel Barquín Alvarez Probleme des Föderalismus, Rolando Tamayo y Salmorán das Verfahren der Verfassungsänderung und Gerardo Gil Valdivia Änderungen des Finanzverfassungsrechts.

Die beiden einzigen Referenten, die nicht aus der iberischen Welt kamen, gingen ebenfalls auf Verfassungsprobleme Mexikos ein: Carl E. Schwartz (USA), der am Instituto de Desarrollo Urbano y Regional in Toluca (Estado de México) tätig

24 Abgedruckt in diesem Heft S. $291 \mathrm{ff}$. 
war, behandelte Fragen der Landes- und Entwicklungsplanung und des Schutzes des Staatsbürgers gegen die Willkür von Behörden. Hans-Rudolf Horn (Bundesrepublik Deutschland) zeigte die Entwicklungslinien des „constitucionalismo social“ in Mexiko auf und machte Verfassungsänderungen auf dem Gebiet des Arbeitsrechts zum Ausgangspunkt weiterführender Betrachtungen über die Funktion sozialer Schutznormen ${ }^{25}$.

Unter den zahlreichen verfassungspolitischen Vorschlägen, die von den Teilnehmern des Kolloquiums vorgetragen wurden, verdient eine Anregung von Diego Valadés Hervorhebung. Sie steht im Zusammenhang mit dem, was bereits über das Problem der "Abdankung des Parlaments“ gesagt wurde (s. o. I.). Das Verbot der Wiederwahl der Abgeordneten für zwei aufeinander folgende Wahlperioden wird oft $\mathrm{zu}$ Unrecht in Verbindung gebracht mit dem Verbot der Wiederwahl des Präsidenten, das eine heute noch im Mittelpunkt des politischen Interesses stehende Forderung der Revolution von 1910 war. In Wirklichkeit wurde es erst 1933 mit der nie verschwiegenen Absicht eingeführt, die Stellung der Parlamentarier als unabhängiger Berufspolitiker zu schwächen. Eine solche Regelung enthielt übrigens auch die Verfassung von Cádiz 1812. Valadés hält die Wiederwahl der Abgeordneten für eine Chance, um die Verbindung des Volkes mit seinen Repräsentanten zu verbessern, da der zwangsläufige Wechsel des Abgeordneten alle drei Jahre dadurch vermieden wird. Es handelt sich hier - was nicht ausdrücklich erwähnt wird - um eine alte Forderung der Oppositionspartei „Partido de Acción Nacional" $(\mathrm{PAN})^{26}$. Die von Valadés geforderte Stärkung der Stellung des Abgeordneten setzt auch eine Verbesserung der wissenschaftlichen Dienste des Parlaments (asesoría técnica) voraus.

\section{Verfassungsrecht und politische Wirklichkeit}

Das unlösbare Dilemma für einen Verfassungsrechtler, der sich mit den politischen Verhältnissen in Lateinamerika auseinandersetzt, besteht darin, daß er in vielen Staaten die wichtigsten Gesetze und Verfassungsänderungen für nichtig halten müßte, wenn er sich rigoros auf den Standpunkt stellt, jedes gesetzgeberische Vorhaben müsse in allen Punkten mit den Verfassungsvorschriften über das Zustandekommen von Gesetzen in Einklang stehen. Sicherlich kann vom juristischen Standpunkt nicht jeder Putsch oder jede gewaltsame Anderung der Regierung als Revolution anerkannt werden, die als Grundlage einer neuen Rechtsentwicklung gelten kann. Wer ohne die erforderlichen Differenzierungen auf die Kritik verzichtet, die bei der Beurteilung von verfassungsrechtlichen Grundsätzen her geboten ist, läuft Gefahr, das böse Wort von dem „derecho constitucional“ als dem „derecho prostitucional“ zu rechtfertigen. Es bedarf keiner Hervorhebung, daß es andererseits unrealistisch wäre, auch Dekrete einer siegreichen Junta, die vernünftige und notwendige gesetzliche Regelungen enthalten, nur deshalb nicht als Rechtsgrundlage anzuerkennen, weil das in der Verfassung vorgeschriebene Gesetzgebungsverfahren nicht im einzelnen eingehalten wurde.

\footnotetext{
25 Deutsche Fassung unter dem Titel „Entwicklungslinien des sozialen Rechtsstaats in Mexiko in Viertel jahresschrift für Sozialrecht 1976, $47 \mathrm{ff}$.

$26 \mathrm{Vgl}$. Luis Calderón Vega, Política y espíritu; compromisos y fugas del cristiano, Morelia (Michoacán) 1965, S. 69, ferner S. 76 ff., 101 ff. Dazu Hans-Rudolf Horn, México: Revolution und Verfassung, 1969, S. 80 .
} 
In aller Regel verzichten Militärregierungen darauf, ihre Vorherrschaft in einer Verfassungsurkunde zu verankern. Unter diesem Gesichtspunkt bedarf die Verfassung von Panama vom 11. Oktober 1972 besonderer Erwähnung. Denn sie schreibt in Artikel 2 die harmonische Zusammenarbeit der Exekutive, Gesetzgebung und Rechtsprechung untereinander und mit den Streitkräften vor. Die Diktatur des Generals Torrijos wird ebenfalls ausdrücklich in den Verfassungstext übernommen. In der Übergangsvorschrift des Artikels 277 heißt es: „Se reconoce como Líder Máximo de la Revolución panameña al General de Brigada Omar Torrijos Herrera, Comandante Jefe de la Guardia Nacional ..." Von großer Bedeutung ist dabei die zeitliche Begrenzung der diktatorischen Befugnisse auf sechs Jahre. Auch in deren näherer Beschreibung liegt ein bemerkenswerter Wille zu einer Selbstbindung und einem Verzicht auf die Willkürherrschaft früherer Caudillos.

Die Einstellung der an dem Kolloquium teilnehmenden Staatsrechtslehrer zur Frage der Intervention der Militärs in den staatlichen Bereich interessierte auch die mexikanische Presse in besonderem Maße. Sie war Gegenstand verschiedener Interviews in Tageszeitungen. Camilo Mena von der Universidad de Ecuador brachte die Meinung mehrerer Kollegen zum Ausdruck, daß eine Einmischung in die Regierungspolitik vom Standpunkt des Verfassungsrechts grundsätzlich abzulehnen sei, daß es aber unvermeidbar sein kann, daß die Offiziere die Regierung übernehmen, um das Land "vor dem Chaos zu bewahren“.

Die neuere politikwissenschaftliche Literatur scheint ebenfalls im Begriffe $z u$ sein, das überkommene allzu simple Bild von dem Militär als dem Wachhund der Bourgeoisie und der Plutokraten zu überwinden, auch wenn es schwierig ist, gegen fest verwurzelte Vorstellungen zu hinterfragen. Die überlieferten Denkkategorien von den stets korrupten und gewalttätigen Militärs, die noch vom Beginn des 19. Jahrhunderts stammen, sind nicht geeignet, die Probleme des 20. Jahrhunderts zu lösen $^{27}$. Die Unhaltbarkeit derartiger Denkschemata, die in manchen Fällen zutreffend sein mögen, wurde spätestens dann deutlich, als die Gleichsetzung von Militärregime und reaktionärer Gewaltherrschaft in immer mehr Fällen durch die Realität links gerichteter Militärjuntas überholt wurde.

Die Probleme der politischen Wirklichkeit können nur dann mit einiger Aussicht auf Erfolg erfaßt werden, wenn die Zusammenarbeit von Verfassungsjuristen und Sozialwissenschaftlern auf eine neue Basis gestellt wird. Wenn es auch zu begrüßen ist, daß der Glaube an eine gleichsam automatische Wirkung einer neuen Verfassung oder eines neuen fortschrittlichen Gesetzes immer mehr, schwindet, wäre es andererseits aber auch verfehlt, die Funktion von Gesetzgebung und Rechtsprechung bei der Herbeiführung gewünschter sozialer Veränderungen $\mathrm{zu}$ unterschätzen ${ }^{28}$ oder gar von dem Juristen zu verlangen, seine traditionelle rechtliche Betrachtungsweise durch die Technik eines Sozialingenieurs zu ersetzen. Man wird demgegenüber gerade auch vom Standpunkt zeitgemäßer Sozial- und Sprachwissenschaften sagen können, daß die Praxis der Gesetzgebung nicht nur in den Industrieländern, sondern auch in vielen Entwicklungsländern Kommunikationstrukturen ausgebildet hat, die geeignet sind, auf gesellschaftliche Veränderungen oder Veränderungsbedürfnisse angemessen $\mathrm{zu}$ reagieren ${ }^{29}$. Aus diesem Grunde lohnt sich

27 Vgl. Herbert Krüger, Militärregime in Ubersee, Beiheft $8 \mathrm{zu}$ VRU.

28 Dazu neigt offenbar Klaus Lenk, Zur instrumentalen Funktion des Rechts bei gesellschaftlichen Veränderungen, VRƯ 1976, 139, bes. $155 \mathrm{f}$.

29 Vgl. Waldemar Schreckenberger, Sozialer Wandel als Problem der Gesetzgebung, Verwaltungsarchiv 1977, 28 (43), wo freilich lediglich die Verhältnisse in der Bundesrepublik erörtert werden; zum Problem des 
weiterhin die Befassung mit dem Verfassungsrecht in besonderem Maße; denn es ist berufen, Grundlagen und Grenzen gesetzgeberischer und politischer Maßnahmen der sozialen Entwicklung zu schaffen. Deren Gehalt und Auswirkungen zu beschreiben, ist Sache einer Politikwissenschaft, die sich an den Vorstellungen und Wünschen des in der Demokratie souveränen Volkes orientiert, ohne sich in demoskopischen Techniken zu erschöpfen ${ }^{30}$.

Die entscheidenden Grundprobleme des Verfassungsrechts und der Verfassungswirklichkeit eignen sich nicht für Diskussionen in isolierten nationalen Bereichen, sondern machen einen internationalen Erfahrungsaustausch unabweislich, der auf der Höhe unserer Zeit ist. In Abwandlung des bekannten Goethe-Wortes könnte man sagen „Mexiko, du hast es besser!“ Hier bedarf es nicht erst einer „Bürgerinitiative gegen rechtswissenschaftliche Provinzialität", wie der Arbeitskreis für überseeische Verfassungsvergleichung genannt wurde ${ }^{31}$. Derartige Anstrengungen wurden unvermeidlich, weil in der Bundesrepublik Deutschland, wie Herbert Krüger hervorgehoben hat, die überseeische Verfassungsvergleichung weder Gegenstand der etablierten Forschung noch amtlicher noch sonstiger öffentlicher Förderung ist. In Mexiko finden die Aktivitäten der UNAM, des Instituto de Investigaciones Jurídicas und des Instituto Iberoamericano de Derecho Constitucional nicht nur die erforderliche Unterstützung und Anerkennung des Staates, der beispielsweise beim internationalen Bodin-Symposion 1976 selbst solche Diskussionen in die Wege leitete, um Probleme des modernen Staates wie das der Souveränität zu behan$\operatorname{deln}^{32}$. Die Rechtsvergleichung findet vielmehr auch beim juristischen Nachwuchs die gebührende Beachtung, weil die Überzeugung besteht, daß es sich nicht um eine exotische Liebhaberei, sondern um Fragen des Berufswissens des Juristen handelt, auf das im Zeitalter der internationalen Verflechtungen zu verzichten untunlich ist $^{33}$.

sozialen Wandels vgl. insbesondere Wolfgang Zapf (Herausgeber), Theorie des sozialen Wandels, 1969, und Wilfried Fiedler, Sozialer Wandel, Verfassungswandel, Rechtsprechung, 1972.

$30 \mathrm{Vgl}$. Georges Burdeau, Einführung in die politische Wissenschaft, 1964, S. 421 ff., bes. S. 425 ff. zur Funktion des Verfassungsrechts und der politischen Parteien.

31 Karl Hernekamp, Uberseeische Verfassungsvergleichung - nicht länger Brachland deutscher Rechtswissenschaft, VRƯ 1976, $291 \mathrm{ff}$.

32 Vgl. dazu den Bericht von Hans-Rudolf Horn, JZ 1977, 197.

33 Zur Bedeutung der Rechtsvergleichung vgl. Héctor Fix-Zamudio in seiner Vorlesung bei der Aufnahme in El Colegio Nacional, die unter dem Titel "Derecho Comparado y ciencia jurídica en México in dessen Veröffentlichungen Band VII, No. 1, S. 233 bis 262, erschien. Der Rang der „ceremonia de toma de posesión $^{\alpha}$ vom 13. November 1974, in deren Verlauf der Vortrag gehalten wurde, wird durch die Gegenwart des Präsidenten der Republik und des Kultusministers verdeutlicht. 


\section{Conclusiones del Primer Congreso Latinoamericano de Derecho Constitucional celebrado en la Ciudad de Mexico del 25 al 30 de agosto}

\section{DECLARACIONES GENERALES}

\section{CONSIDERANDO}

Primero: Que el problema fundamental de los pueblos de la América Latina estriba en la honda diferencia de niveles de vida que separa a las clases sociales y en la existencia de grandes masas de trabajadores y campesinos sumidas en la miseria;

Segundo: Que los pueblos están moralmente obligados al establecimiento de una igualdad real entre los hombres, que suprima la miseria y asegure a todos los seres humanos una existencia decorosa;

Tercero: Que el Derecho Constitucional no puede ser un estatuto meramente formal, que estructure los poderes públicos y determine las bases y garantías de su equilibrio, sino que debe ser el ordenamiento mediante el cual se cumplan los postulados de la justicia social;

Cuarto: Que únicamente en un mundo en el jue exista una igualdad real y en el jue se haya desterrado la miseria, pueden florecer las libertades fundamentales para todos los seres humanos;

\section{DECLARA :}

Es una de las finalidades fundamentales del Derecho Constitucional contribuir a la creación de una sociedad orientada hacia la justicia social, en la Uue desaparezca la miseria y se establezca una igualdad, que asegure a todos los seres humanos una existencia decorosa, que les permita desarrollar plenamente sus aptitudes y disfrutar de las libertades esenciales del hombre y de los beneficios de la civilización y de la cultura.

El Primer Congreso Latinoamericano de Derecho Constitucional

$$
\text { CONDENA: }
$$

todos los regímenes latinoamericanos, de los que es trágico ejemplo el caso de Chile, que nacidos del avasallamiento y menosprecio de la Constitución, violando los derechos de la persona humana y poniéndose al servicio de las oligarquías, se sustentan sólo en el ejercicio ilegítimo de la fuerza y se apoyan y vinculan a los grupos de presión internacionales.

\section{RECOMENDACIONES Y CONCLUSIONES}

\section{TEMA I}

El Predominio del Poder Ejecutivo en Latinoamérica

Primera.-La democracia ha de ser el sustento de los regímenes constitucionales de nuestros pueblos que luchan por decidir su destino.

Segunda.-El Derecho Constitucional se explica en función de factores políticos, económicos, sociales y culturales.

Tercera.-El predominio del Poder Ejecutivo en América Latina es, en consecuencia, el resultado de esos factores y del proceso histórico que la vivido nuestro Continente. Cuarta.-El sistema presidencial latinoamericano, por su origen y por su evolución, posee características propias que lo apartan del modelo norteamericano. Entre los aspectos en que se manifiesta esa divergencia hay que destacar el principio de la no reelección del titular del Ejecutivo - sostenido por la mayoría de nuestros paísesprincipio que supone el carácter temporal del ejercicio del poder.

Quinta.-Es necesario distinguir el sistema presidencial sujeto al orden constitucional, del caudillismo, la dictadura y otras formas patológicas del predominio del Poder Ejecutivo en América Latina.

Sexta.-El Derecho Constitucional latinoamericano debe contener los sistemas que garanticen la efectividad del equilibrio de los poderes. El cambio de las estructuras económico-sociales y el desarrollo cultural y político de nuestros pueblos contribuirán a la efectividad de ese principio.

Séptima.-La complejidad de la vida contemporánea y las necesidades de la política del desarrollo de los pueblos latinoamericanos, exigen un Poder Ejecutivo con las facultades constitucionales suficientes para encararlas $y$ resolverlas. El Poder Ejecutivo se legitima en la constitución que es el límite que la razón del hombre opone a la arbitrariedad, ha de fundarse en la mayoría popular y defender los derechos de la persona humana.

Octava.-Las atribuciones del Ejecutivo deben controlarse mediante eficaces mecanismos legislativos y jurisdiccionales. Es conveniente estudiar la posible adopción de nuevas formas democráticas de control del Poder Ejecutivo. 
Es indispensable y urgente reforzar la educación política, uno de cuyos efectos será el que la opinión pública - elemento fundamental de la democracia - llegue a ser un efectivo control del Poder Ejecutivo. Han de garantizarse, en consecuencia, los derechos de libertad de expresión, de manifestación pública del pensamiento, de difusión de las ideas a través de los medios de comunicación y la libertad de las universidades como factores decisivos de formación de la opinión pública.

Novena.-El debilitamiento del sistema federal en los países en donde existe, ha contribuido al predominio del Ejecutivo latinoamericano; por lo mismo, dicho sistema federal ha de ser replanteado, adecuándolo a las necesidades del desarrollo de nuestros pueblos.

Décima.-Es conveniente que, según las características de cada país, se analice la posibilidad de separar la posición de líder o jefe del partido oficial de la función de titular del ejecutivo, incluso por prohibición constitucional o legal.

\section{TEMA II}

Constitución y Grupos de Presión

Primera.-El Congreso observa que la tendencia moderna del derecho constitucional de Latinoamérica se dirige a reconocer y reglamentar la existencia y el funcionamiento de los grupos intermedios, conjuntamente con las garantías individuales, en tanto esos grupos sean instrumentos de protección de intereses colectivos y de participación para el desarrollo de la vida comunitaria.

Segunda.-La imposibilidad del individuo, en cuanto tal, para defenderse contra la violación de intereses colectivos o grupales, hace necesario autorizar y promover la organización y participación de grupos sociales para la protección colectiva de los individuos y de los derechos de la comunidad.

Tercera.-Debido a los riesgos que son inherentes a la formación de grupos intermedios, cuando actúan como grupos de presión ilegítima, y a la posible deformación de la estructura, objetivos y fines de los mismos, es necesario establecer, incluso a nivel constitucional, la reglamentación de principios democráticos que rijan el funcionamiento de dichos grupos.

Cuarta.-Son condenables las actividades directas u ocultas de los grupos de presión, nacionales, regionales o internacionales, tendientes a perturbar o alterar el orden jurídico constitucional de América Latina y a establecer regímenes políticos antidemocráticos productos de golpes de Estado, putch militares, golpes de astucia, cooptación y presidencia vitalicia o hereditaria, frecuentemente propiciados por los intereses egoístas de los grupos de presión.

Quinta.-Exhortar a que en los estudios constitucionales latinoamericanos se consideren los modelos de Estado marxista y fascista.

\section{TEMA III}

Cambios Constitucionales en América Latina

\section{PRIMERA PARTE}

Primera.-Es prioritario definir el modelo de organización constitucional que se requiere para cada país, pues de él dependerá la dimensión de las funciones públicas y, por consiguiente, inmediatamente después, o a partir de ello, se podrán definir las demás características del nuevo régimen.

Segunda.-Dentro de ese orden de ideas, es necesario conocer, a través de la Constitución, la distribución de competencias entre el sector público y el sector privado, ya que de las normas que incorpore la constitución surgirán o no, nuevas funciones y órganos estatales.

Tercera.-La Constitución no debe convertirse en una ley reglamentaria ni incurrir en un excesivo detallismo, sino que, en la generalidad de sus normas, deben encontrarse los lineamientos fundamentales para que los órganos competentes instrumenten una organización adecuada.

Cuarta.-Además de las competencias conferidas a los poderes estatales, es conveniente que la constitución contenga una o más cláusulas programáticas dirigidas a orientar a los gobernantes políticos en la dirección de los fines que el Estado debe realizar e indicar a los órganos de interpretación y control, cuál es el sentido y la ideología de la constitución.

Quinta.-En los países de régimen federativo, debe acudirse las enseñanzas que emanan del derecho público provincial (estadual), toda vez que la más reciente 
modernidad de las constituciones provinciales permite extraer de sus normas, al tiempo de la sancion de las constituciones nacionales, pero que ofrecen la ventaja contenidos ejemplares que, por obvias razones cronológicas, no fueron previsibles de su incorporación a la experiencia del derecho positivo a nivel local.

Sexta.-En el mismo orden de ideas, son rescatables diversas prescripciones incorporadas a los antecedentes constitucionales latinoamericanos y a los proyectos elaborados durante las últimas décadas, que deben ser tenidos en cuenta a los efectos de las reformas próximas; como asimismo, otras enseñanzas extraídas de las más modernas constituciones de otros continentes por la vía del método comparativo.

Séptima.-Cualquier regulación constitucional que se intente, déberá cuidar la armonia entre la protección del plano en que se desenvuelven los derechos subjetivos e intereses legítimos, por una parte, y la no interferencia, que asegure la plena funcionalidad operativa de los poderes y organismos previstos para el cumplimiento de los fines estatales consagrados en la Constitución, por la otra.

Octava.-El equilibrio entre los diversos planos en juego, debe complementarse a nivel normativo, con el aprovechamiento de las conclusiones elaboradas por la ciencia política y la ciencia de la administración, para la mejor organización del sector público en todos sus aspectos $\mathrm{y}$, a la vez, con un adecuado sistema de controles, que permitan, en su conjunto, proteger razonablemente las notas de eficiencia Y libertad que debe procurar todo ordenamiento.

Novena.-Las materias que pueden ser objeto de regulación constitucional, requieren un tratamiento metodológico conducente a una adecuada sistematización en el futuro texto constitucional, de manera tal, que la diversidad de temas, encuentre sus respectivos agrupamientos lógicos, dando lugar incluso, a la incorporación de nuevos capítulos o títulos específicos (v. gr., sobre "administración pública", "régimen económico", etc.)

Decima.-Las normas a incorporar a las constituciones también admiten clasificación conforme a la consagrada distinción de las cláusulas constitucionales, en: a) organizativas, en cuanto se refieren a las competencias y organismos que deben cumplir funciones del Estado; b) preceptivas, en cuanto se refieren a los principios, límites y garantías que dan protección a la actividad de los administrados; y c) programáticas, en cuanto envuelven directivas orientadas a los gobernantes políticos y al compromiso que vincula a la Constitución con sus intérpretes y demás autoridades de aplicación. Undécima.-En el estudio de los cambios y reformas constitucionales, como, en general, de cualquier problema de derecho constitucional no debemos constreñirnos al estudio de la constitución escrita.

La validez de la constitución escrita, como la cualquiera otra legislación, se encuentra condicionada por su eficacia. Es por ello que, cuando la legislación constitucional ha dejado de ser efectiva, es necesario preguntarse por el derecho que existe en la comunidad política y no contentarse con señalar, la existencia de una "situación de facto".

A la ineficacia de la legislación constitucional no corresponde un "vacio jurídico". La ineficacia de la legislación constitucional supone, necesariamente, un comportamiento jurídico e institucional en contrario.

Duodécima.- Ante la ineficacia de la constitución escrita, es menester determinar las disposiciones y prescripciones que funcionan como la constitución de la comunidad política. Tales disposiciones serán resultado del proceso consuetudinario que, paralelamente a la desaplicación e inobservancia de la constitución escrita, produce un derecho constitucional consuetudinario.

Décimotercera.- En virtud de que las reformas a la constitución pueden contener elementos contrarios al espíritu o a la letra de la propia carta suprema, introduciendo normas que pudieran considerarse, desde el punto de vista del sistema, inconstitucionales, es conveniente que se generalice el procedimiento de consulta popular para reformar la constitución en cuestiones de especial importancia.

\section{SEGUNDA PARTE}

Primera.-Es conveniente que las constituciones latinoamericanas establezcan el derecho de todo hombre al acceso a una propiedad privada capaz de satisfacer sus necesidades esenciales para una vida y un hogar dignos.

Segunda.- Las constituciones latinoamericanas debieran establecer como regla general, que en caso de conflicto entre el interés general y el interés particular, aquél prevalece sobre éste, y como consecuencia de ello, que la ley puede imponer el derecho de propiedad las limitaciones que reclame el interés social.

Tercera.-Es de toda conveniencia que las constituciones latinoamericanas indiquen y delimiten las nuevas funciones económicas que asume el Estado en la vida actual, sea 
como planificador de las actividades económicas nacionales, sea como regulador imperativo de ellas, sea como gestor directo de ciertas industrias básicas o de servicio de utilidad pública. En este último caso, debe precisarse que estas labores las puede cumplir directamento o al través de entidades estatales o paraestatales creadas con tal fin.

Cuarta.-Los preceptos constitucionales latinoamericanos deben dar cabida a la nacionalización como un instituto jurídico diferente de la expropiación clásica y sometida a reglas diversas de esta última. Mediante la nacionalización se expresa la soberanía del Estado, con objeto de traspasar a la propiedad colectiva, empresas de producción o distribución de bienes que la conveniencia pública exige sean explotadas por la nación.

Quinta.-Entre las reglas especiales sobre nacionalización que deben ser introducidas en las constituciones latinoamericanas está la de que la indemnización que por esa nacionalización se pague, queda confiada, en cuanto a su monto y forma de pago, las leyes y reglamentos internos del Estado que nacionaliza. Igualmente las controversias que esa indemnización suscite, deberán ser resueltas por los tribunales del Estado que nacionaliza y con arreglo a sus leyes.

\section{TEMA IV}

La Función del Poder Judicial en los Sistemas Constitucionales Latinoamericanos

\section{PRIMERA PARTE}

Primera.-Es necesaria una mayor aproximación entre los constitucionalistas y los cultivadores del procesalismo científico, con el objeto de estudiar, con mayor profundidad y en forma integral, las materias que comprenden las zonas de confluencia entre ambas disciplinas, y que tienen relación directa con la función del organismo judicial.

Segunda.-Resulta indispensable la reafirmación de la función política del organismo judicial, entendida como intervención técnica para determinar el alcance y el espíritu de las normas constitucionales, la que asume particular dificultad en el ámbito latinoamericano debido a la existencia de organismos ejecutivos predominantes, $y$, en algunos casos, dictatoriales; y esta función se centra en el control judicial de la constitucionalidad de las leyes reconocida por casi todas las constituciones de Latinoamérica, pero, además, en la interpretación directa o indirecta de las disposiciones supremas y la tutela de los derechos humanos consagrados en la carta fundamental.

Tercera.-Si se quiere lograr la realización práctica de la justicia constitucional en América Latina, deben adoptarse una serie de reformas del organismo judicial entendido en un sentido integral y de conjunto, y para ello debe otorgarse a los tribunales administrativos cualquiera que sea su encuadramiento formal, todas las facultades imperativas predominantes de la función judicial, incluyendo la de ejecutar coactivamente sus resoluciones contrarias a la administración; darle independencia al ministerio público, respecto del ejecutivo, separando las atribuciones de asesoría y de representación del gobierno de las de representación social y persecución de los delitos, ya que esta última requiere de autonomía; $\mathrm{y}$, finalmente, configurar la abogacia latinoamericana con un nuevo espíritu de servicio social, que le permita abandonar su papel tradicional de profesionista liberal, para asumir la de colaboración efectiva con el juzgador.

Cuarta.-La función jurisdiccional de los tribunales latinoamericanos, especialmente las cortes supremas y el ejercicio de la función de justicia constitucional, sólo podrá lograr efectividad, así sea limitada, cuando se superen los obstáculos hasta ahora insalvables de la interpretación desorbitada de los actos políticos y de gobierno, así calificados con razón o sin ella, de revolucionarios, y para ello, podrían utilizarse los instrumentos de la "razonabilidad" que ha sostenido valientemente en alguna época la Suprema Corte Argentina, y en lo que resulte aplicable, el recurso de "desvió de poder", creador por la jurisprudencia del Consejo de Estado francés para determinar los límites de las facultades discrecionales del ejecutivo.

Quinta.-En la realización del control de la constitucionalidad de las leyes, los tribunales latinoamericanos deben superar el principio adoptado por razones históricas, de la desaplicación concreta de la ley, para consignar el de declaración general de inconstitucionalidad tomando en cuenta las particularidades y experiencias de cada régimen jurídico, con el objeto de darle verdadera eficacia práctica.

Sexta.-En la reorganización del aparato judicial latinoamericano, resulta conveniente la introducción del consejo judicial o de la magistratura, el cual deberá ser organizado teniendo en cuenta las características peculiares de la realidad constitucional latino- 
americana. Debe estar integrado mayoritariamente por funcionarios judiciales, cuya designación provenga de los mismos jueces, sin perjuicio de la representación que el organismo judicial se transforme en un cuerpo cerrado y estratificado. Su función será la de vigilar el cumplimiento de las garantías judiciales, especialmente el nombramiento, promoción y responsabilidad de los jueces y el establecimiento de carreras Y política judiciales.

Séptima.-El organismo judicial debe tener facultades colegislativas, es decir, poder presentar iniciativas ante el órgano legislativo de los ordenamientos relacionados con su función, y también la de formular dictámenes respecto a los proyectos que sobre esta materia redacten otras autoridades.

Octava.-Debe insistirse en la necesidad de lograr la verdadera autonomía financiera de los tribunales latinoamericanos, y el sistema más adecuado es el de consagrar en el texto constitucional un porcentaje mínimo del presupuesto nacional.

Novena.-Debe combatirse hasta donde esto sea posible, las dos afecciones patológicas de la función judicial: la lentitud y la onerosidad procesales. Puesto que especialmente desvirtúan la tutela de los derechos fundamentales y el control de la constitucionalidad de las leyes así como los derechos de los justicables, también constitucionales, de la acción de la defensa en juicio; para ello es preciso actualizar nuestros viejos códigos procesales, establecer un mínimo de oralidad, y reorganizar la judicatura.

Décima.-El acceso a la jurisdicción como derecho fundamental de los justiciables no se alcanza de manera efectiva con los sistemas tradicionales, todavía practicados en Latinoamérica, de la justicia gratuita, el patrocinio voluntario de los abogados; el beneficio de pobreza o la defensoría de oficio; sino que debe estudiarse la posibilidad de adoptar, además, con las debidas precauciones, los nuevos instrumentos de la asesoría legal establecida recientemente en varios países, que han llegado a concebir dicho asesoramiento como una institución de justicia social.

Undécima.-Debe modificarse la tradicional pasividad de los jueces latinoamericanos, otorgándoles facultades de dirección del proceso, y la colaboración de una abogacía independiente dotada de espíritu de justicia social. Ello implica una serie de reformas que deben partir de una transformación de la enseñanza del derecho y el establecimiento de especialización de las diversas profesiones jurídicas.

Duodécima.-Que se estimule la expansión internacional del amparo, especialmente entre los países signatarios de la Declaración de Bogotá que todavía no lo han adoptado.

\section{SEGUNDA PARTE}

\section{CONSIDERANDO}

Primero.-Que en todos los tiempos y en todos los países los jueces ejercen una actividad que "latu sensu" puede Ilamarse política en el sentido de que cuando aplican las leyes las interpretan según el espíritu del tiempo en que viven.

Segundo.-Que tan política es su función cuando, como en la mayor parte de los países latinoamericanos, los jueces están llamados también a controlar la conformidad de las leyes con las constituciones de sus respectivos países.

Tercero.-Que, por lo demás, se manifiesta hoy la necesidad de la participación de los jueces en una función promocíonal que se está convirtiendo en típica dentro de cada ordenamiento progresivo, dado que todos contienen normas de estímulo y de incentivo como las que se dan en el campo de la salud del patriminio histórico y artístico y de la cultura, en general.

CONCLUYE

Primero.-Que es necesario un mayor acercamiento del juez a la base social, especialmente mediante un intercambio recíproco de información entre la magistratura y la opinión pública que permita la libre crítica de su actividad aún por parte de los que no son técnicos en derecho.

Segundo.-Que el juez debe interpretar las normas a aplicar a la luz de los principios constitucionales aprovechando la elasticidad que poseen y siguiendo los cánones histórico evolutivos, para que, sin perjuicio de respetar al máximo la regla de la certeza del derecho, puedan contribuir al desarrollo y progreso moral y civil de la sociedad moderna en su rápida evolución. 


\section{TERCERA PARTE}

Pririera.-El amparo mexicano, con su existencia más que centenaria, ha servido de modelo a las instituciones de amparo de diversos países latinoamericanos, que lo han incorporado a su régimen constitucional con las modalidades propias de cada uno de ellos.

Segunda.-El amparo mexicano, en su procedencia y teleología genéricas subsume los objetivos de los recursos similares que operan en diversos países latinoamericanos para la protección de los gobernados contra actos del poder público del Estado.

Tercera.-El amparo mexicano, según tradicionalmente ha sido desde que se creó por don Manuel Crescencio Rejón en la Constitución yucateca de diciembre de 1841, configura un proceso constitucional, en el que se manejan conceptos comunes a todo proceso.

Cuarta.-Dada la existencia más que secular del amparo mexicano, este ha ido asumiendo una conceptuación y una terminología propias.

Quinta.-El amparo mexicano, sin detrimento de las excelencias que puedan tener los recursos similares existentes en otros países latinoamericanos, es el mejor medio jurídico-procesal de rango constitucional, de que dispone todo gobernado para defender su esfera jurídica frente a cualquier acto de autoridad que provenga de cualquier órgano del Estado.

Sexta.-Es deseable que el amparo mexicano se haga expresamente procedente contra actos de entidades paraestatales, como ya acontece en varios países latinoamericanos. Séptima.-Los catos de individuos particulares que afectan la esfera jurídica de otros particulares no deben hacer procedente el amparo ya que existen otros medios jurídicos para hacer cesar las infracciones que dichos actos produzcan y para reparar los daños o agravios que causen. 